\title{
Unusual Closed Traumatic Avulsion of Both Flexor Tendons in Zones 1 and 3 of the Little Finger
}

\author{
Marie-Aimée Päivi Soro, Thierry Christen, and Sébastien Durand \\ Department of Plastic and Hand Surgery, Lausanne University Hospital, rue du Bugnon 46, 1011 Lausanne, Switzerland \\ Correspondence should be addressed to Marie-Aimée Päivi Soro; paivi.soro@gmail.com
}

Received 29 May 2016; Accepted 11 August 2016

Academic Editor: Johannes Mayr

Copyright (c) 2016 Marie-Aimée Päivi Soro et al. This is an open access article distributed under the Creative Commons Attribution License, which permits unrestricted use, distribution, and reproduction in any medium, provided the original work is properly cited.

\begin{abstract}
Closed tendon avulsion of both flexor tendons in the same finger is an extremely rare condition. We encountered the case of a patient who presented a rupture of the flexor digitorum profundus in zone 1 and flexor digitorum superficialis in zone 3 in the little finger. This occurrence has not been reported previously. We hereby present our case, make a review of the literature of avulsion of both flexor tendons of the same finger, and propose a treatment according to the site of the ruptures.
\end{abstract}

\section{Introduction}

Closed tendon avulsion is a well recognized injury in hand surgery. Also called Jersey finger, it usually involves the flexor digitorum profundus (FDP) tendon. The typical mechanism is a forced hyperextension on a fully flexed finger. It is often encountered in contact sports like rugby, American football, or judo when a player grabs his opponent's shirt with the tip of his finger while the opponent is running away.

Closed avulsion of the flexor digitorum superficialis (FDS) associated with an avulsion of the FDP is a rare occurrence. Only 8 cases have been reported since 1984 [1-9].

We encountered an unusual case of closed avulsion of both flexor tendons of the little finger with a rupture of the FDP in zone 1 and FDS in zone 3. Combination of simultaneous avulsion in zones 1 and 3 has not been reported previously. We will present our case, review the literature, and propose a treatment according to the site of the ruptures.

\section{Case Report}

A 30-year-old patient presented to the emergency unit after a rugby game with the impossibility to flex his fifth finger on the left hand. Surgical exploration was performed the same day; it demonstrated a rupture of the FDP a few millimetres from its insertion and a laceration of the FDS in the mid-palm (Figure 1). No previous trauma or other pathology could explain this double rupture.
The FDP was reinserted using a pull-out technique. In order to avoid flexion deformity of the distal interphalangeal (DIP) joint related to excessive tension of the FDP, the procedure was completed with a lengthening Z-plasty of the FDP in the forearm proximal to the carpal tunnel.

As the rupture of the FDS occurred in the mid-palm, a reconstruction would not interfere with the course of the FDP in the digital canal. Therefore the suture of the FDS was reinforced with a palmaris longus tendon graft.

The patient was immobilized in a Duran splint and underwent the usual rehabilitation protocol for flexor tendon lesions with physical therapists.

Follow-up at 6 months showed the patient was able to touch his palm with his little finger (Figures 2 and 3 ) and return to work. The mobility of the finger in extension/flexion was as follows: metacarpophalangeal (MCP) joint: $0 / 0 / 95^{\circ}$, proximal interphalangeal (PIP) joint: $0 / 10 / 85^{\circ}$, and distal interphalangeal (DIP) joint: $0 / 10 / 25^{\circ}$. Active flexion of the PIP joint was observed when action of the FDP was prevented.

\section{Discussion-Review of the Literature}

We found 8 case reports (11 fingers) of closed avulsion of both flexor tendons in a single finger [1-9]. The characteristics of the patients, injury type, surgical repair technique, and results are summarized in Table 1. 


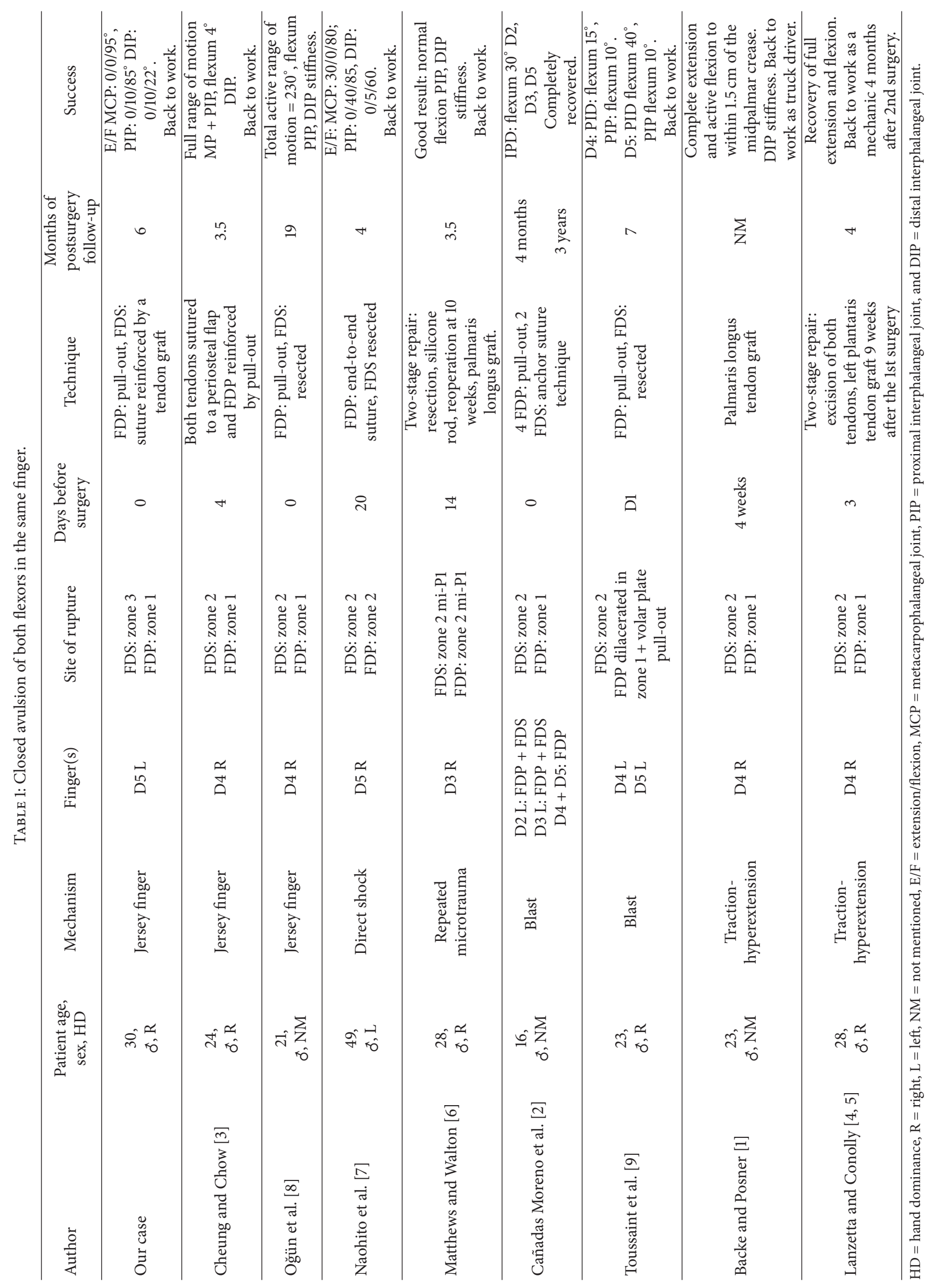




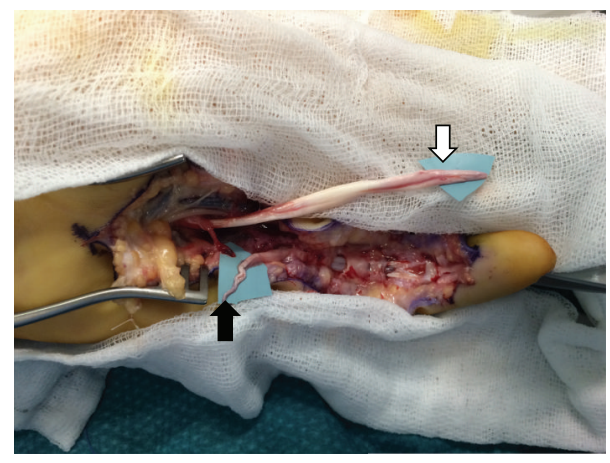

FIGURE 1: The white arrow indicates the proximal stump of the FDP, and the black arrow the distal dilacerated stump of the FDS.

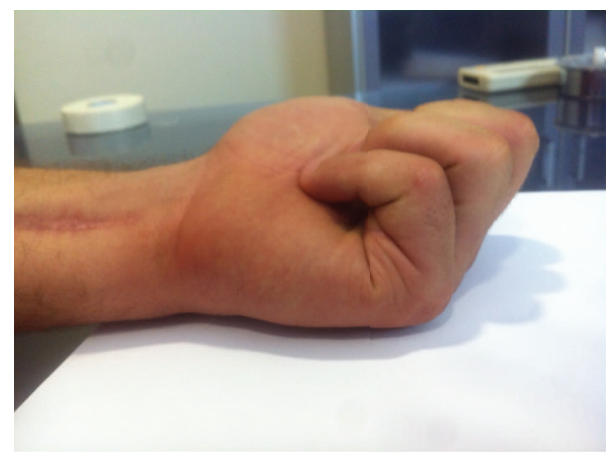

FIGURE 2: 6 months after surgery.

The mean age of the patients was 26.9 years. They were all men. The dominant hand was affected in $42 \%$ of patients (three authors did not mention the hand dominance). The ring finger (5/11 fingers) was the most commonly affected. Six different mechanisms of injury were encountered. Two patients presented with a typical Jersey finger [3, 8]. Two others suffered a blast injury [2,9] and had several fingers affected. Two patients had a mechanism of tractionhyperextension $[1,4,5]$. The patient from Naohito et al. [7] hurt his fifth finger in a fall without further details. Repeated microtraumas were responsible for the rupture in one case [6]. The mean interval between traumatism and surgical exploration was 7.8 days (median 3 days). Five patients underwent surgery in the first week after trauma and 3 patients after 2 weeks.

One author used ultrasonography as a diagnostic help [9]. Their localisation of the rupture was accurate.

All FDS tendons were ruptured in zone 2 except in our case (zone 3).

All FDP including our case were ruptured in zone 1 except for two in zone $2[6,7]$.

Six authors chose to resect the FDS [1,4-9]. In the other two cases [2,3], the rupture was not intratendinous but at the insertion and associated with a bony avulsion. Both authors reinserted it with a transosseous suture.

Four authors reinserted the FDP through a pull-out suture [2, 3, 8, 9]. Naohito et al. [7] performed an end-to-end suture for a zone 2 rupture. Three authors $[1,4-6]$ performed

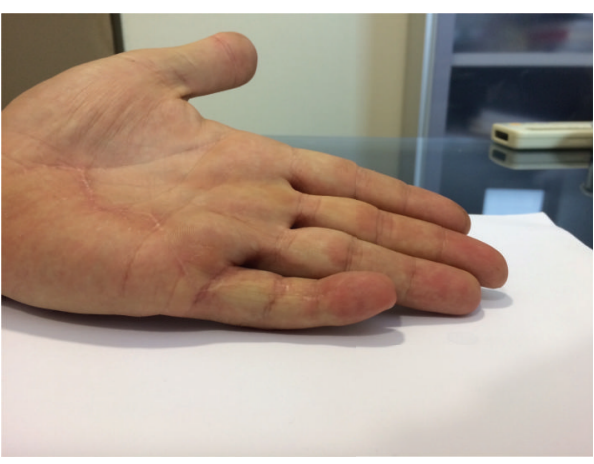

FIGURE 3: 6 months after surgery.

an FDP resection followed by a tendon graft in a one- or twostage procedure when rupture was in zone 2 or in cases of delayed presentation.

The outcome was reported as good to excellent in 4 cases out of 8 . In the other four cases, the results were poorer due to an FDP lesion in zone 2 or dilacerated in zone 1 . Seven out of nine patients went back to work with no or little disability [1, $3-7,9]$. Two authors did not mention whether their patients were able to return to normal activity $[2,8]$.

Our case is unusual as the FDS was dilacerated in zone 3. The literature shows that few patients ( 2 out of 8 ) benefited from an FDS repair $[2,3]$. This is probably due to the fact that both flexor tendon sutures in zone 2 are known to result in poorer outcome than more proximal lesions [10]. The rupture in zone 3 allowed for an FDS suture reinforced by a tendon graft. The other distinctive characteristic that our case presents is that the FDP pull-out suture was completed with a lengthening Z-plasty in the forearm which prevented tension deformity from the DIP and PIP joint. We were able to repair both tendons and avoid a suture in zone 2 . The result was good at the 6-month follow-up visit and the patient was able to return to work.

Only one author used ultrasonography as a diagnostic tool before surgery [9]. Its utility has been shown as a diagnostic help for flexor tendon injuries [11, 12]. Double flexor tendon injury in the same finger is a rare occurrence and the usefulness of ultrasonography still needs to be explored in this indication. However, it might be an interesting tool to assess the zones in which the tendons have ruptured in order to define preoperatively surgical strategy.

In conclusion:

(1) When FDS is ruptured in zone 3 and FDP in zone 1, an FDS repair is recommended as it will not impair the FDP function in the digital canal and reinforces the action of the FDP. The FDP can benefit from a pullout suture. If the FDP is dilacerated and the pull-out suture prevents full extension of the DIP or PIP joint we recommend a lengthening Z-plasty in the forearm so as to avoid a resection-graft which has worse outcomes.

(2) When the FDS is ruptured in zone 2 and the FDP in zone 1 , we recommend to reinsert the FDP with 
a pull-out suture. An FDS reinsertion could be considered in particular conditions $[2,3]$.

(3) If both tendons are ruptured in zone 2 , whenever possible, both tendons should be repaired, as long as the FDP is gliding freely. If the tendons are severely dilacerated or oedematous and the FDS suture impairs the FDP course in the digital canal, the FDS should be resected [10]. The FDP, depending on the dilacerations and extension of the lesions, could be either sutured or grafted $[6,7]$. Another surgical option is the transfer of a hemi-FDP-tendon from an adjacent finger [13].

\section{Competing Interests}

The authors declare that they have no competing interests.

\section{Acknowledgments}

The authors warmly thank Miss Robin Avona Hampton for her careful read-through of our manuscript.

\section{References}

[1] H. Backe and M. A. Posner, "Simultaneous rupture of both flexor tendons in a finger," The Journal of Hand Surgery, vol. 19, no. 2, pp. 246-248, 1994.

[2] O. Cañadas Moreno, R. Martínez González-Escalada, and F. J. Lara García, "Multiple closed avulsions of flexor tendons of the hand caused by a firecracker blast," Annals of Plastic Surgery, vol. 68, no. 2, pp. 158-160, 2012.

[3] K. M. C. Cheung and S. P. Chow, "Closed avulsion of both flexor tendons of the ring finger," Journal of Hand Surgery, vol. 20, no. 1, pp. 78-79, 1995.

[4] M. Lanzetta and W. B. Conolly, "Simultaneous rupture of both flexor tendons in a finger," The Journal of Hand Surgery, vol. 21, no. 6, pp. 1114-1115, 1996.

[5] M. Lanzetta and W. B. Conolly, "Biomechanical explanation of a simultaneous closed rupture of both flexor tendons in the same digit," Australian and New Zealand Journal of Surgery, vol. 66, no. 3, pp. 191-194, 1996.

[6] R. N. Matthews and J. N. Walton, "Spontaneous rupture of both flexor tendons in a single digit," The Journal of Hand Surgery, vol. 9, no. 2, pp. 134-136, 1984.

[7] H. Naohito, A. Masato, A. Rui, H. Daisuke, Y. Yusuke, and I. Koichi, "Closed rupture of both flexor digitorum profundus and superficialis tendons of the small finger in zone II: case report," Journal of Hand Surgery, vol. 36, no. 1, pp. 121-124, 2011.

[8] T. C. Oğün, H. M. Ozdemir, and H. Senaran, "Closed traumatic avulsion of both flexor tendons in the ring finger," The Journal of Trauma, vol. 60, no. 4, pp. 904-905, 2006.

[9] B. Toussaint, E. Lenoble, O. Roche, C. Iskandar, J. Dossa, and Y. Allieu, "Subcutaneous avulsion of the flexor digitorum profundus and flexor digitorum superficialis tendons of the ring and little fingers by blast injury," Annales de Chirurgie de la Main et du Membre Supérieur, vol. 9, no. 3, pp. 232-235, 1990.

[10] J. B. Tang, "Flexor tendon repair in zone 2C," The Journal of Hand Surgery, vol. 19, no. 1, pp. 72-75, 1994.

[11] T. S. Sügün, N. Karabay, T. Toros, K. Özaksar, M. Kayalar, and E. Bal, "Validity of ultrasonography in surgically treated zone 2 flexor tendon injuries," Acta Orthopaedica et Traumatologica Turcica, vol. 44, no. 6, pp. 452-457, 2010.

[12] S. B. Cohen, A. B. Chhabra, M. W. Anderson, and M. E. Pannunzio, "Use of ultrasound in determining treatment for avulsion of the flexor digitorum profundus (rugger jersey finger): a case report," American Journal of Orthopedics, vol. 33, no. 11, pp. 546549, 2004.

[13] S. Durand, C. Oberlin, and A. MacQuillan, "FDP to FDP hemitendon transfer-a new technique for delayed repair of the flexor digitorum profundus in zones I and II of the finger," The Journal of Hand Surgery, vol. 35, no. 8, pp. 677-678, 2010. 


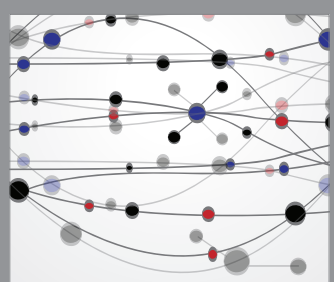

The Scientific World Journal
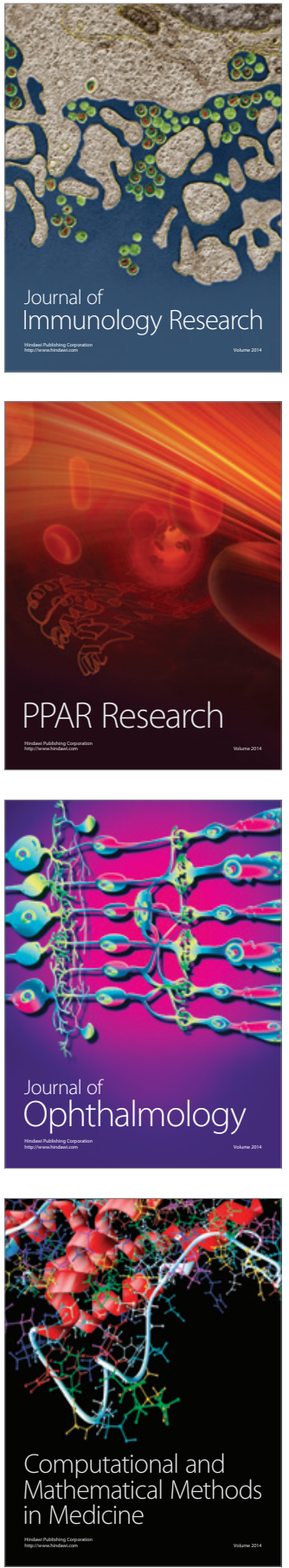

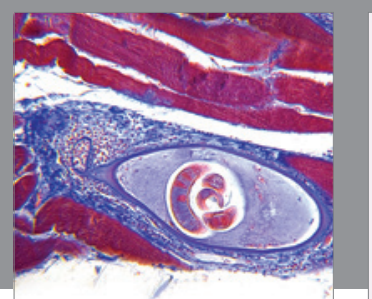

Gastroenterology Research and Practice

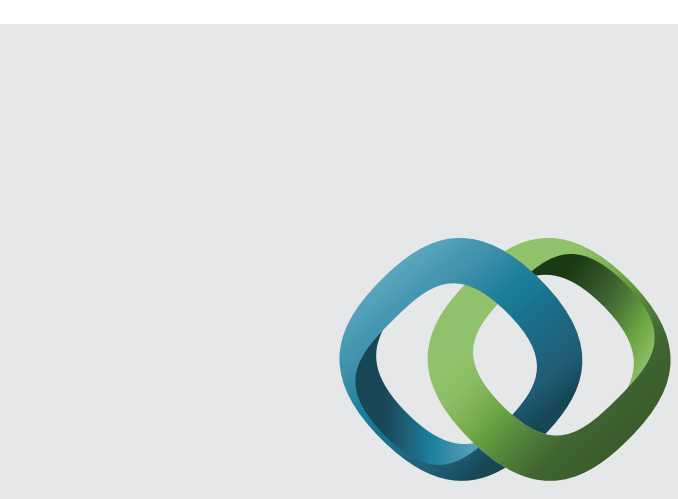

\section{Hindawi}

Submit your manuscripts at

http://www.hindawi.com
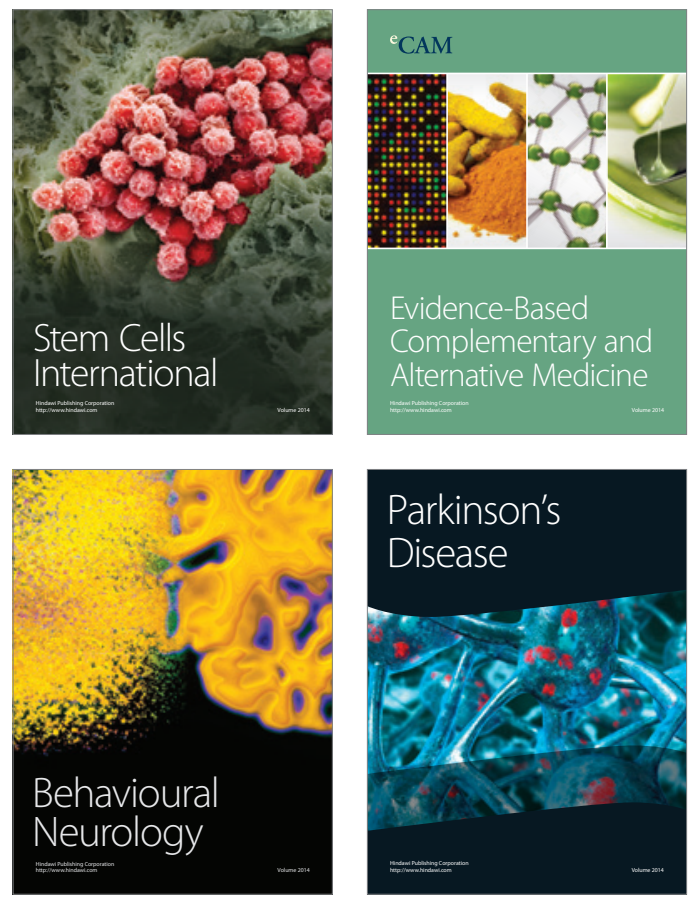
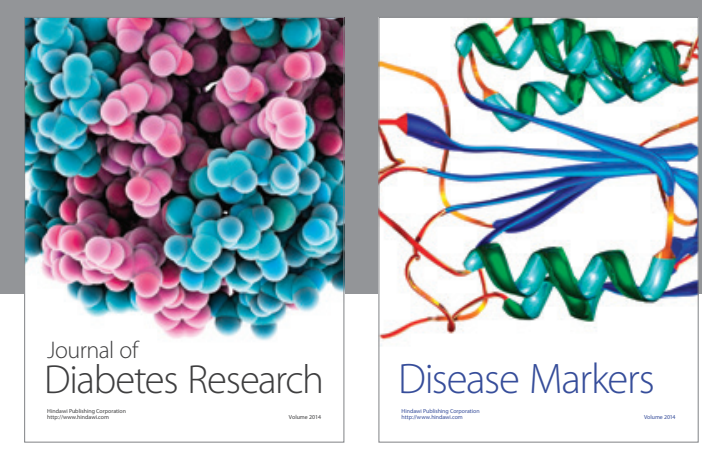

Disease Markers
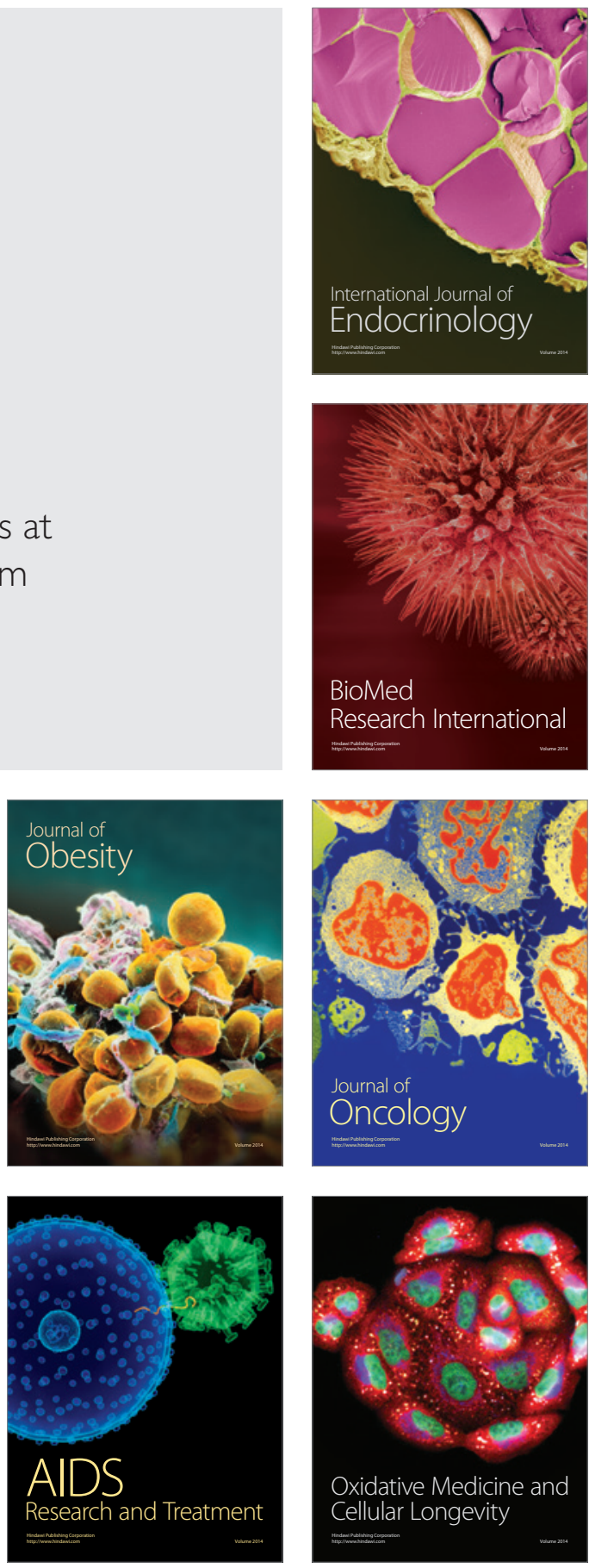